\title{
Simulation du préformage de renforts composites textiles 3D
}

\author{
Emmanuel de Luycker ${ }^{1}$, Philippe Boisse $^{1, a}$, Fabrice Morestin $^{1}$, David Marsal $^{2}$ \\ ET StÉPhane OTIN ${ }^{2}$ \\ 1 Laboratoire de Mécanique des Contacts et des Structures LaMCoS (UMR CNRS 5259) $18-20$ rue des sciences, \\ INSA-Lyon, 69621 Lyon, France \\ 2 Département Matériaux YQMM, Snecma Villaroche, Réau, rond point R. Ravaud, 77550 Moissy-Cramayel, France
}

Reçu le 12 juillet 2010, accepté le 12 juillet 2010

Résumé - Ce travail s'inscrit dans le cadre de la simulation de la mise en forme de matériaux composites. Le cas traité ici est celui du préformage des interlocks. Il s'agit de renforts textiles 3D permettant d'éviter le délaminage rencontré avec des stratifiés. Un élément-fini 3D semi-discret permettant de modéliser la déformation d'une préforme interlock complète est décrit. Chaque élément tridimensionnel contient des fibres qui contribuent à la rigidité de l'élément. Les paramètres matériaux sont identifiés à partir d'essais mécaniques et la simulation est validée par comparaison avec l'expérience.

Mots clés : Tissés / textiles 3D / interlocks / propriétés mécaniques / éléments-finis (MEF) / mise en forme

\begin{abstract}
D textile composite reinforcement preforming simulations. This work is a contribution to the simulation of composite forming. The performing of interlock reinforcement is considered in the present paper. They are 3D textile reinforcements that avoid the delamination that can occur in the case of laminate composites. A 3D semi-discrete finite element that allows to model the deformation of an interlock perform is described. Each three-dimensional element contains some fibres that contribute to the element stiffness. The material parameters are identified from mechanical tests and the simulation is validated by comparison with experiment.
\end{abstract}

Key words: Fabrics / textiles 3D / Interlocks / mechanical properties / finite-element analysis (FEA) / forming

\section{Introduction}

Les composites, qui présentent un rapport résistance/masse souvent avantageux, sont de plus en plus utilisés notamment dans l'industrie aéronautique. Ce développement est également favorisé par la diminution du prix des matières premières et la maîtrise de procédés permettant des coûts de production limités tels que le RTM (resin transfer molding) au cours duquel la résine est injectée sur un renfort sec préformé dans un moule [1-3]. Pour les pièces de forte épaisseur, les interlocks [4], illustrés figure 1, peuvent être utilisés. Ces matériaux tissés dans l'épaisseur permettent d'éviter les délaminages rencontrés dans les composites de type stratifiés tout en facilitant l'élaboration des pièces finies en évitant l'empilement d'un nombre important de plis.

\footnotetext{
a Auteur pour correspondance :

Philippe.Boisse@insa-lyon.fr
}

Leur application à des pièces structurelles telles que les aubes de soufflante de moteurs d'avions Snecma représentées figure 1 est ainsi possible.

Dans ce contexte, la maîtrise des propriétés dynamiques et de tenue à l'impact du matériau fini est indispensable. Le comportement du composite étant très influencé par la direction des fibres, une connaissance fine du préformage présente donc un intérêt certain. La simulation numérique de ce préformage permet d'accéder à la mésostructure du composite fini (taux volumique de fibres, position et orientation des mèches, angle de cisaillement entre les réseaux) et permet également de quantifier la faisabilité d'une mise en forme (apparition de plis ou de flambements, efforts sur les outils, ruptures de fibres). Les données issues du préformage (taux de fibres, cisaillement) sont aussi précieuses pour évaluer la perméabilité du matériau et ainsi prévoir la phase d'injection (apparition de zones sèches ou de zones pauvres en fibres). 

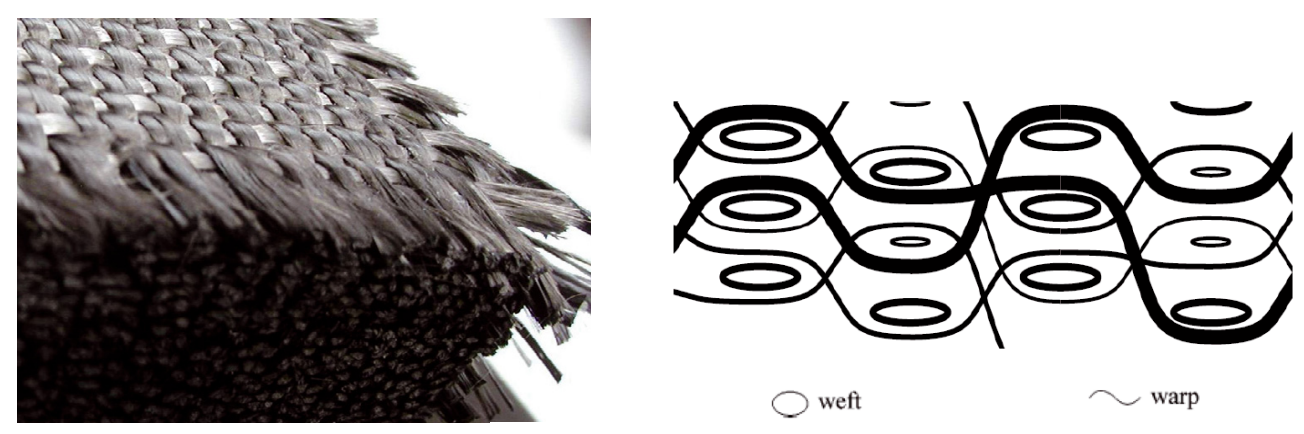

$\frown$ warp

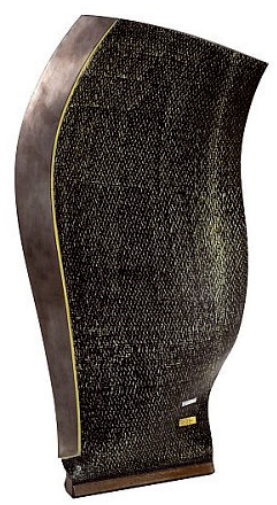

Fig. 1. Tissé interlock 3D/aube de soufflante Snecma à renfort interlock.

\section{Simulation du préformage de renforts interlocks}

Plusieurs méthodes existent pour la simulation de renforts 2D, s'appuyant sur des approches continues ou discrètes [5-8]. En 3D, pour les pièces considérées, aucune de ces deux approches n'est satisfaisante car une prise en compte des contacts entre mèches conduit à des coûts de calcul prohibitifs et la non périodicité du matériau conduit à des difficultés grandes transformations en terme d'homogénéisation. Néanmoins, la mésostructure initiale est connue puisque nécessaire au tissage de la préforme. Le comportement macroscopique d'un matériau est influencé par sa microstructure et sa mésostructure. Nous proposons d'utiliser l'information de position de chaque mèche dans le textile pour rendre compte de son anisotropie. Pour cela, un élément-fini semi-discret dédié aux matériaux interlocks illustré figure 2 est introduit. Le comportement global de l'interlock est découpé en deux contributions, l'une modélisée de manière discrète, issue des mèches en tension, l'autre par un modèle continu, issue des autres mécanismes.

\section{1 Écriture spécifique de l'équation de la dynamique}

Le comportement mécanique d'un tissé 3D interlock est très spécifique. La raideur d'une mèche constituée de milliers de fibres (généralement entre $3 \mathrm{~K}$ et $48 \mathrm{~K}$ fibres) est principalement une rigidité de tension dans la direction tangente à la mèche. Le très faible diamètre des fibres (entre $5 \mu \mathrm{m}$ et $20 \mu \mathrm{m}$ pour le carbone, l'aramide et le verre) et leur mouvement relatif possible confèrent à la mèche des rigidités de flexion, cisaillement et compression transverse très faibles devant la rigidité de tension. Les déformations du matériau pendant le préformage sont alors principalement dues aux tensions dans les mèches. Ceci doit évidemment être nuancé car dans certaines directions, la rigidité due aux tensions peut être négligeable et dans ce cas, les autres rigidités deviennent prépondérantes.

Dans le principe des travaux virtuels, nous distinguons alors pour les travaux virtuels intérieurs et pour les efforts internes (dans une discrétisation élément-finis), un terme de tension $W_{\text {int }}^{\text {t* }}$ et un terme provenant des autres contributions $W_{\text {int }}^{\mathrm{a} *}$.

$$
\begin{aligned}
& W_{\mathrm{ext}}^{*}-\left(W_{\mathrm{int}}^{\mathrm{t} *}+W_{\mathrm{int}}^{\mathrm{a} *}\right)=W_{\mathrm{acc}}^{*} \\
& W_{\mathrm{int}}^{\mathrm{t} *}+W_{\mathrm{int}}^{\mathrm{a} *}=\mathbf{U}^{* \mathrm{~T}} \mathbf{F}_{\mathrm{int}}=\mathbf{U}^{* \mathrm{~T}}\left(\mathbf{F}_{\mathrm{int}}^{\mathrm{t}}+\mathbf{F}_{\mathrm{int}}^{a}\right)
\end{aligned}
$$

Les termes de tension conduisant à des efforts internes prépondérants étant donnée la raideur élevée des mèches en tension, la géométrie de chaque mèche est prise en compte pour permettre le calcul du comportement anisotrope de la structure. Un modèle discret est utilisé à ce niveau alors que les autres contributions sont prises en compte par un modèle continu isotrope. 

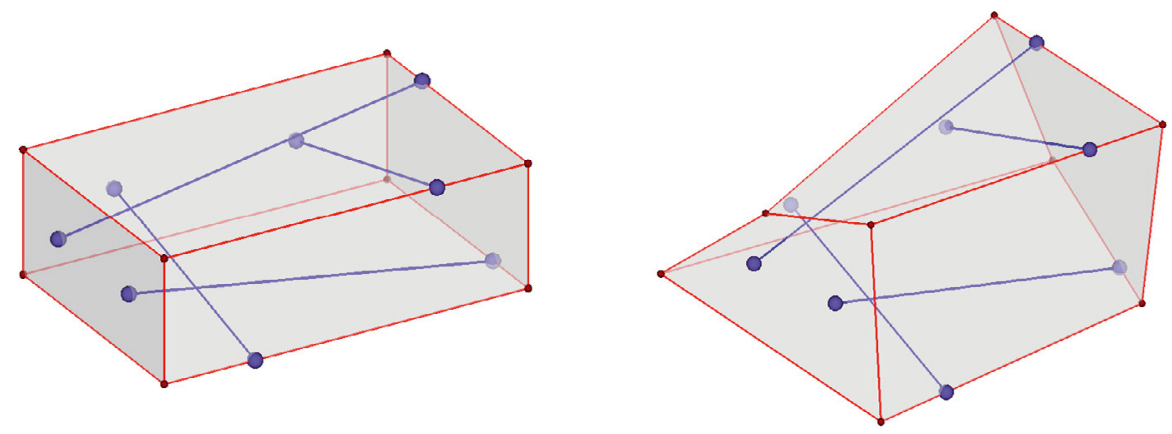

Fig. 2. Élément-fini semi-discret constitué de mèches. Avant et après déformation.
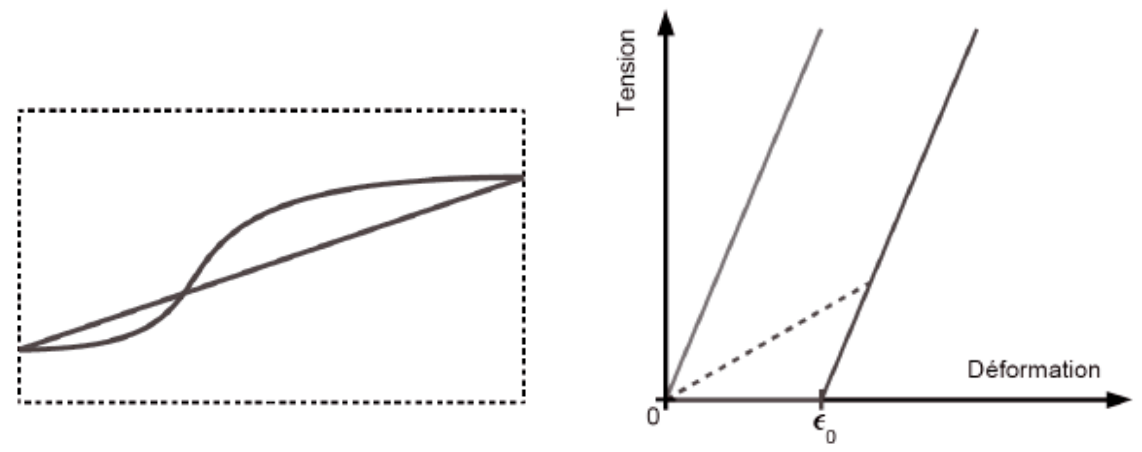

Fig. 3. Prise en compte de l'ondulation de la mèche dans le comportement en tension.

\section{3 Élément-fini spécifique}

\section{1 Élément-fini constitué de segments de mèches}

La préforme interlock 3D est maillée avec des éléments hexaédriques tels que celui représenté figure 2. Des segments de mèches sont ajoutés en calculant l'intersection des mèches réelles avec chaque élément. Nous utilisons un élément à huit nœuds avec des fonctions de forme tri-linéaires. Par cohérence avec cette interpolation, les mèches sont prises rectilignes à l'intérieur de l'élément, l'ondulation éventuelle d'une mèche dans l'élément étant prise en compte par une modification du comportement de chaque mèche. L'élément est lagrangien. Ainsi, la position des mèches reste inchangée dans l'espace de référence et leur cinématique est déterminée à partir des déplacements nodaux de l'élément. La position de chaque mèche dans chaque élément est déterminée à partir des spécifications de la préforme nécessaires à son tissage (trajet spatial de chaque mèche).

\subsection{Efforts internes de tension}

Les mèches sont modélisées par des segments droits. Le travail virtuel de tension développé à l'intérieur de la mèche $p$ est l'intégrale sur la longueur de mèche $L^{p}$ du produit de la tension $T^{p}$ avec la déformation axiale virtuelle dans la direction de la mèche $\varepsilon^{p}\left(\underline{u}^{*}\right)$ :

$$
W_{\mathrm{int}}^{\mathrm{tp}}\left(\underline{u}^{*}\right)=\int_{L^{p}} T^{P} \varepsilon^{p}\left(\underline{u}^{*}\right) \mathrm{d} L
$$

Après discrétisation élément-fini, le vecteur des efforts internes est obtenu par sommation des contributions des $N^{\mathrm{m}}$ mèches contenue dans l'élément. L'intégration est réalisée à partir de deux points de Gauss sur chaque mèche.

$$
W_{\text {int }}^{\mathrm{te}}\left(\underline{u}^{*}\right)=\sum_{p=1}^{\mathrm{N}^{\mathrm{m}}} \int_{L^{p}} T^{P} \varepsilon^{p}\left(\underline{u}^{*}\right) \mathrm{d} L=\mathbf{U}^{\mathrm{eT}} \mathbf{F}_{\mathrm{int}}^{\mathrm{te}}
$$

Le calcul de la déformation virtuelle est réalisé par suivi des vecteurs covariants de l'élément et projection sur le vecteur normé tangent à la mèche comme détaillé dans [9] La tension est déterminée à partir d'une raideur de mèche et de la déformation cumulée. Pour prendre en compte une ondulation de mèche dans l'élément, le comportement de la mèche est pris non-linéaire. Cette non-linéarité représentée figure 3 est déterminée à partir de la différence de longueur de la mèche réelle (ondulée) et du segment rectiligne qui la représente.

\subsection{Efforts internes des autres contributions - modèle hypoélastique}

La majeure partie de la rigidité du renfort interlock provient des mèches en tension, ce sont elles qui engendrent l'anisotropie caractéristique des tissés. Néanmoins, d'autres mécanismes entrent en jeu dans la mécanique des textiles tels que la compression transverse des mèches, le cisaillement ou encore la friction entre les différents éléments. Ces dernières contributions restant de 
second ordre un modèle hypoélastique isotrope est utilisé pour les modéliser. Ce type de modèle est largement utilisé dans les codes éléments finis pour modéliser le comportement isotrope de matériaux continus en grandes transformations [10-12]. Si des travaux existent concernant l'extension de ces modèles aux matériaux anisotropes $[2,13,14]$, on suppose ici que le comportement correspondant aux contributions complémentaires est isotrope. L'anisotropie est liée à la partie fibre. Cette approche hypoélastique est directement extensible à la plasticité $[12,15,16]$ ce qui est utile si l'on veut prendre en compte les frottements inter mèches $[17,18]$.

Une dérivée objective $\underline{\sigma}^{\mathrm{a} \nabla}$ du tenseur des contraintes de Cauchy est calculée à $\overline{\bar{p}}$ artir du taux de déformation $\underline{\underline{D}}$ :

$$
\underline{\underline{\sigma}}^{\mathrm{a} \nabla}=\underline{\underline{\underline{\underline{C}}}}^{\mathrm{a}}: \underline{\underline{D}}
$$

L'exposant a fait référence aux autres contributions, complémentaires de celles des mèches en tension. $\underline{\underline{\underline{C}}}^{\mathrm{a}}$ est le tenseur de comportement élastique isotrope. Ce comportement est modifié pour tenir compte de la spécificité des matériaux fibreux qui sont très compressibles, et le module de Young est modifié pour traduire un raidissement en fonction du taux volumique au sein de chaque élément. Le coefficient de Poisson est quant à lui pris nul, ce qui correspond à la réalité pour ce type de matériau, au moins pour de faibles compactions. L'objectivité du taux de contrainte est assurée en utilisant un repère lié à la matière. Dans le cas présent, nous utilisons la dérivée de Jaumann qui suit la rotation moyenne de la matière. Le cumul de contrainte est ensuite réalisé grâce au schéma d'intégration de Hughes et Winget [10] à partir de l'incrément de contrainte calculé.

$$
\left[\sigma^{\mathrm{a} n+1}\right]_{\mathrm{e}_{i} n+1}=\left[\sigma^{\mathrm{a} n}\right]_{\mathrm{e}_{i}^{n}}+\left[\mathbf{C}^{\mathrm{a} n+1 / 2}\right]_{\mathrm{e}_{i}^{n+1 / 2}}[\Delta \varepsilon]_{\mathrm{e}_{i}^{n+1 / 2}}
$$

La base orthonormée ei est déterminée par la rotation du repère corotationnel à partir de la base locale. L'incrément de déformation est calculé à partir du pas de temps et du taux de déformation exprimé à partir des matrices d'interpolation des déformations B standard des hexaèdres à huit nouds.

$$
\Delta \varepsilon=\mathbf{D}^{n+1 / 2} \Delta t=\mathbf{B}\left(\dot{\mathbf{u}_{n}^{e}}\right)^{n+1 / 2} \Delta t
$$

Les efforts nodaux élémentaires relatifs aux autres contributions sont calculés par :

$$
\mathbf{F}_{\text {int }}^{\mathrm{ae}}=\int_{\mathrm{V}^{\mathrm{e}}} \mathbf{B}^{\mathrm{T}} \sigma^{\mathrm{a}} \mathrm{d} V
$$

\section{Validation de l'élément et identification des paramètres}

\subsection{Tests élémentaires de validation de l'élément}

L'élément est implémenté dans un code en dynamique explicite développé au LaMCoS. Une série de tests
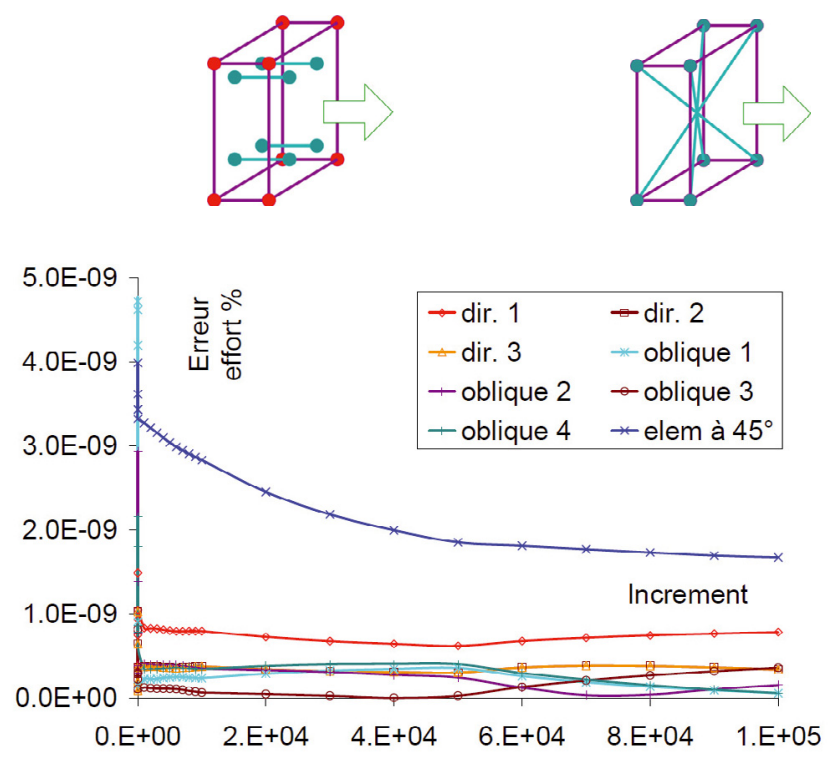

Fig. 4. Erreur sur un élément en traction à déplacements imposés pour différentes orientations des mèches.

élémentaires pour différentes orientations de mèches, à déplacements imposés et à efforts imposés permet de valider l'élément (Fig. 4). D'autres tests élémentaires sont détaillés dans $[9,19]$.

\subsection{Identification des paramètres du modèle}

\subsubsection{Raideur des mèches en tension}

La raideur des mèches en tension est utilisée dans le calcul des efforts nodaux de tension (Éq. (3)). Deux méthodes peuvent être envisagées pour son identification. Tout d'abord cette raideur en tension peut être calculée à partir du nombre de fibres par mèche et de leur section. Connaissant le module de Young du carbone et supposant un comportement linéaire d'une fibre on obtient la raideur d'une mèche. Il convient de noter que dans les applications, les préformes contiennent des mèches de sections très différentes. Cette raideur est donc propre à une mèche ou plutôt une famille de mèches. Il convient ensuite de tenir compte de l'ondulation de la mèche dans l'élément considéré (Fig. 3).

Une autre approche consiste à réaliser un test de traction sur une éprouvette dont les mèches étudiées sont dans la direction de traction. On obtient une courbe telle que celle présentée figure $6 \mathrm{a}$. La première partie non-linéaire est liée aux ondulations. La deuxième partie linéaire permet de déterminer la raideur en tension des mèches. Les valeurs obtenues par cette approche sont plus faibles que celles calculées à partir de la raideur de chaque fibre [9]. Il est probable que dans un ensemble complexe de mèches et fibres tel qu'une éprouvette en interlock épais, toutes les fibres ne sont pas tendues exactement de façon similaire dans un essai de traction, ce qui explique la valeur plus faible obtenue expérimentalement pour la raideur en tension. 

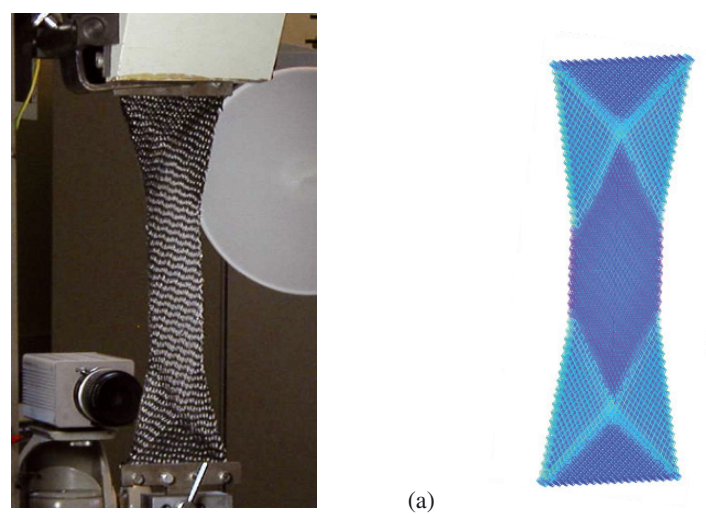

(a)

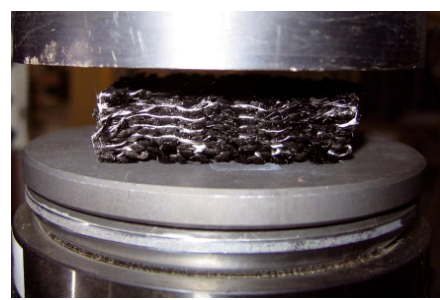

(b)

Fig. 5. Essai de cisaillement bias extension test (a) et essai de compression (b) sur un interlock.

\subsubsection{Identification du modèle hypoélastique}

Le comportement hypoélastique a pour objectif d'apporter les rigidités autres que celles liées à la tension des mèches. Le modèle est supposé isotrope à coefficient de Poisson nul. Deux essais sont utilisés pour déterminer le module de Young. Celui-ci est fortement fonction de l'état des déformations. Il est supposé dépendre du taux volumique de fibres. Ce module de Young fixe le module de cisaillement puisque le comportement est supposé isotrope. Deux essais sont utilisés : l'essai de cisaillement de bias extension test [20] réalisé par traction d'une éprouvette dont les mèches sont à $\pm 45^{\circ}$ par rapport à la direction de sollicitation (Fig. 5a) et un essai de compression transverse (Fig. 5b). Le module de Young est identifié en fonction de la fraction volumique de fibre par approche inverse, grâce à un algorithme d'optimisation de type LevenbergMarquardt [21] (Figs. 6b et c). Ces deux identifications conduisent à des valeurs de module de Young (fonction de la fraction volumique de fibres) qui sont en accord de façon acceptable.

\section{Simulations du préformage d'un renfort 3D interlock et validation expérimentale}

Un interlock est mis en forme sur un hémisphère. Cette forme présente une double courbure et ce formage nécessite un cisaillement dans le plan important. Une série d'essais est réalisée expérimentalement pour des préformes interlock de différentes épaisseurs (Fig. 7a). Les angles de cisaillement sont mesurés par stéréo corrélation $[22,23]$. Ils sont différents sur les faces

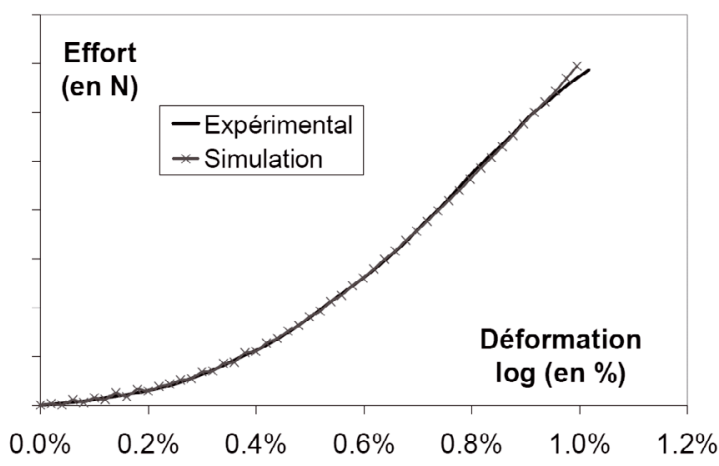

(a) Traction

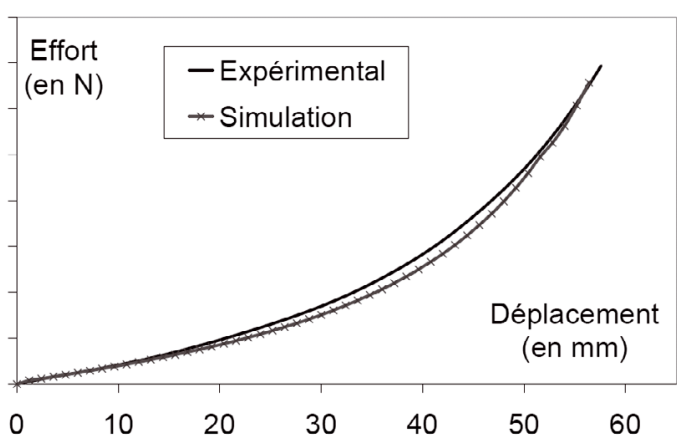

(b) Cisaillement Bias Test

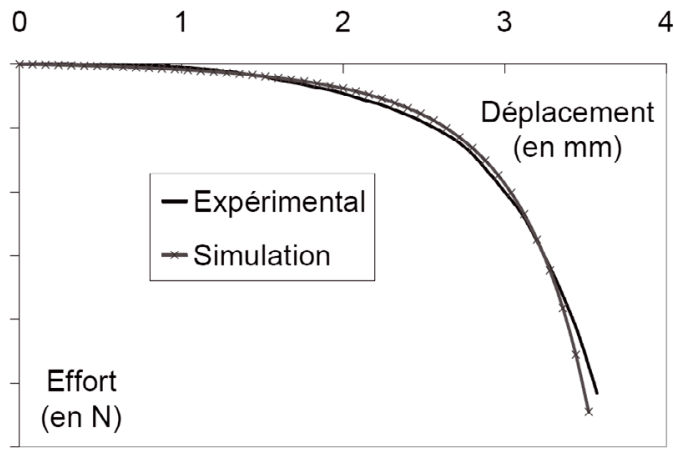

(c) Compression

Fig. 6. Identifications : de la raideur en tension (a); du modèle hypoélastique en cisaillement (b) et compression (c).

interne et externe de l'hémisphère et sont en bon accord avec les simulations (Fig. 7b) [9].

\section{Conclusion}

Un élément-fini hexaédrique constitué de segments de mèches est proposé pour la simulation du préformage de renforts $3 \mathrm{D}$ interlocks. La position de chaque mèche est prise en compte de manière discrète alors que les propriétés transverses sont modélisées par un modèle continu hypoélastique. Le modèle semi-discret a été validé et identifié sur des cas tests et des essais mécaniques 


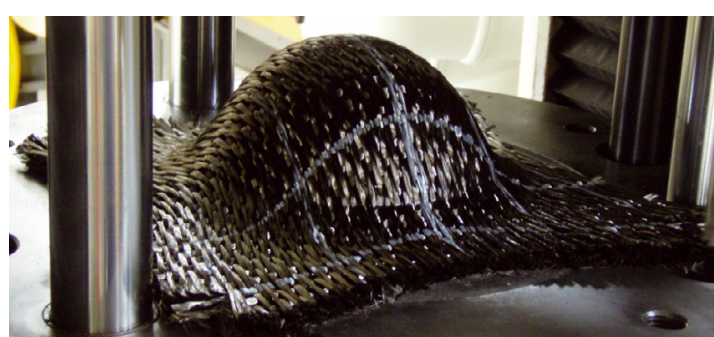

(a)

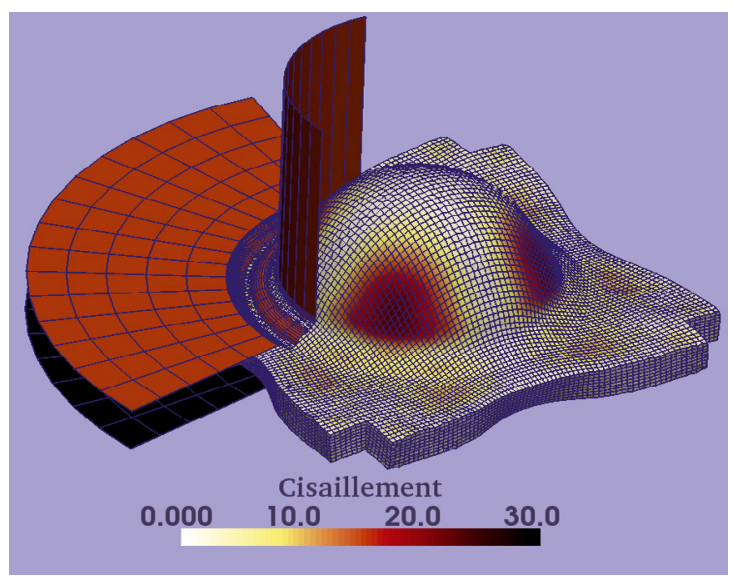

(b)

Fig. 7. Simulation d'emboutissage hémisphérique d'un interlock. Expérimentation (b) simulation.

standard. Il a montré sa pertinence par comparaison essai/simulation d'emboutissages hémisphériques.

Remerciements. Les auteurs remercient la Société SNECMA pour son soutien technique et financier. Ce travail s'inscrit dans le projet fédérateur MAIA de recherche et technologie en mécanique soutenu par le CNRS, l'ONERA et le groupe SAFRAN. Ce travail entre pour partie dans le cadre des projets ANR LCM3M et MECAFIBRES.

\section{Références}

[1] R.S. Parnas, Liquid Composite Molding, Hanser Garner publications, 2000

[2] S.G. Advani, Flow and rheology in polymeric composites manufacturing, Elsevier, Amsterdam, 1994

[3] A. Saouab, J. Bréard, P. Lory, B. Gardarein, G. Bouquet, Injection simulations of thick composite parts manufactured by the RTM process, Compos. Sci. Tech. 61 (2001) $445-451$

[4] A.P. Mouritz, M.K. Bannister, P.J. Falzon, K.H. Leong, Review of applications for advanced three-dimensional fibre textile composites, Composites Part A 30 (1999) 1445-1461

[5] X. Peng, J. Cao, A continuum mechanics-based nonorthogonal constitutive model for woven composite fabrics, Composites Part A 36 (2005) 859-874

[6] P. Boisse, B. Zouari, J.L. Daniel, Importance of In-Plane Shear Rigidity in Finite Element Analyses of Woven Fabric Composite Preforming, Composites Part A 37 (2006) 2201-2212
[7] R.H.W. Ten Thije, R. Akkerman, J. Huetink, Large deformation simulation of anisotropic material using an updated Lagrangian finite element method, Comput. Methods Appl. Mech. Eng. 196 (2007) 3141-3150

[8] G. Hivet, P. Boisse, Consistent 3D geometrical model of fabric elementary cell. Application to a meshing preprocessor for 3D finite element analysis, Finite Elem. Anal. Des. 42 (2005) 25-49

[9] E. De Luycker, Thèse de doctorat de l'INSA de Lyon, 2009

[10] T.J.R. Hughes, J. Winget, Finite rotation effects in numerical integration of rate constitutive equations arising in large deformation analysis, Int. J. Numer. Methods Eng. 15 (1980) 1862-1867

[11] M.A. Criesfield, Non linear Finite Element Analysis of Solids and Structure : Advanced Topics, John Wiley, Chichester, 1997, Vol. 2

[12] T. Belytschko, K.L. Wing, B. Moran, Nonlinear Finite Elements for Continua and Structures, John Wiley, Chichester, 2000

[13] V.G. Oshmyan, S.A. Patlazhan, Y. Remond, Principles of Structural-Mechanical Modeling of Polymers and Composites, Polymer Science Ser. A 48 (2006) 1004-1013

[14] P. Badel, S. Gauthier, E. Vidal-Sallé, P. Boisse, Rate constitutive equations for computational analyses of textile composite reinforcement mechanical behaviour during forming. Composites: Part A 40 (2009) 997-1007

[15] D. Peric, On consistent stress rates in solid mechanics : Computational implications, Int. J. Num. Meth. Eng. 33 (1992) 799-817

[16] M.L. Boubakar, P. Boisse, J.C. Gelin, Numerical implementation of orthotropic plasticity for sheet metal forming analysis, J. Mater. Process. Technol. 65 (1997) 143152

[17] V. Carvelli, A. Talierico, A micromechanical model for the analysis of unidirectional elastoplastic composites subjected to 3D stresses, Mech. Res. Commun. 26 (1999) $547-553$

[18] S.V. Lomov et al., Meso-FE modelling of textile composites : road map, data flow and algorithms, Compos. Sci. Tech. 67 (2007) 1870-1891

[19] E. De Luycker, F. Morestin, P. Boisse, D. Marsal, Simulation of 3D interlock composite preforming, Compos. Struct. 88 (2009) 615-623

[20] J. Cao et al., Characterization of mechanical behavior of woven fabrics: Experimental methods and benchmark results, Composites Part A 39 (2008) 1037-1053

[21] D.S. Schur, N. Zabaras, An inverse method for determining elastic material properties and a material interface, Int. J. Numer. Methods Eng. 33 (1992) 2039-2057

[22] F. Dumont, G. Hivet, R. Rotinat, J. Launay, P. Boisse, P. Vacher, Identification des caractéristiques mécaniques de renforts tissés à partir de mesures de déformations par corrélation d'images, Mécaniques \& Industries 4 (2003) 627-635

[23] S.V. Lomov, P. Boisse, E. De Luycker, F. Morestin, K. Vanclooster, D. Vandepitte, I. Verpoest, A. Willems, Fullfield strain measurements in textile deformability studies, Composites: Part A 39 (2008) 1232-1244 